\title{
The first seroepidemiological survey for Angiostrongylus vasorum in domestic dogs from Romania
}

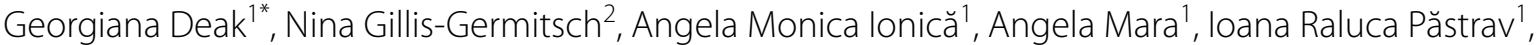 \\ Cristina Daniela Cazan ${ }^{1}$, Mariana Ioniță³ , Ioan Liviu Mitrea ${ }^{3}$, Cristian Răileanu ${ }^{4,5}$, Diana Bărburaș ${ }^{1}$, \\ Maria Nedișan ${ }^{1}$, Răzvan Oachiș ${ }^{6}$, Vasile Cozma', Roland Schaper ${ }^{7}$, Manuela Schnyder $^{2}$ \\ and Andrei Daniel Mihalca' ${ }^{1}$
}

\begin{abstract}
Background: Angiostrongylus vasorum is a metastrongyloid nematode localized in the right heart and the pulmonary arteries of domestic dogs. The number of reports in Europe has recently increased, presumably as a consequence of a growing awareness among clinicians, animal owners and researchers, but also due to a growing incidence and territorial spread. So far, no studies have been conducted to assess the prevalence and distribution of $A$. vasorum in domestic dogs in Romania, and the awareness among veterinarians is limited or absent. The aim of the present study was to evaluate the countrywide seroprevalence of circulating antigens of $A$. vasorum and specific antibodies against A. vasorum in domestic dogs from Romania.
\end{abstract}

Methods: Between November 2016 and July 2017, blood was sampled from a total of 1545 domestic dogs from 23 counties of Romania. Details about their gender, age, breed, housing, use and origin were collected. All serum samples were tested for the presence of $A$. vasorum circulating antigens (AG) using monoclonal and polyclonal antibodies in a sandwich ELISA. Additionally, a sandwich ELISA using A. vasorum adult somatic antigen purified by monoclonal antibodies was used for specific antibody (AB) detection.

Results: A total of 33 dogs (2.14\%, 95\% Cl: 1.82-3.56\%) were seropositive for A. vasorum antigen or antibodies against the parasite. Three dogs were positive for antigen only $(0.19 \%, 95 \% \mathrm{Cl}: 0.07-0.57 \%)$ and 30 dogs $(1.94 \%, 95 \% \mathrm{Cl}$ : $1.36-2.76 \%$ ) were positive for antibodies only. No dog was positive for both tests. The overall prevalence (AB or AG) and the $A B$ prevalence were significantly higher in pure breed dogs compared to mixed breeds and mongrel dogs $(P<0.05)$ and in shepherd dogs compared to other groups $(P<0.05)$. There was no significant difference between males and females, between urban and rural dogs, between dogs with unrestricted access and with restricted access to the environment, and between dogs living outdoors and indoors.

Conclusions: Our data suggest that the disease is present in Romania in dogs, as it was previously demonstrated in foxes. However, so far, no clinical case has been reported in the country and this may be related to a low awareness among vets.

Keywords: Canine angiostrongylosis, Romania, Angiostrongylus vasorum, Serology, Antigen and antibody detection

\footnotetext{
*Correspondence: georgiana.deak@usamvcluj.ro

1 Department of Parasitology and Parasitic Diseases, University of Agricultural Sciences and Veterinary Medicine Cluj-Napoca,

Cluj-Napoca, Romania

Full list of author information is available at the end of the article
} 


\section{Background}

Angiostrongylus vasorum is a metastrongyloid nematode localized in the right heart and the pulmonary arteries of mainly domestic dogs [1] and foxes (Vulpes vulpes) [2], but also in other wild carnivores: gray wolves (Canis lupus) [3], golden jackals (Canis aureus) [4], coyotes (Canis latrans) [5], red pandas (Ailurus fulgens) [6], meerkats (Suricata suricatta) [7] and various mustelids, using mainly gastropods as intermediate hosts $[8,9]$ and possibly amphibians and birds as paratenic hosts [10]. Infection of definitive hosts may also occur by ingestion of vegetation, food or water contaminated with secretions from infected gastropods $[1,11]$.

Since its first description in 1853 in France [12], $A$. vasorum is presently considered to have a wide distribution and lately the number of reports in Europe has increased presumably as a consequence of a growing awareness among clinicians, owners and researchers, but also due to a growing incidence and territorial spread [13].

Angiostrongylus vasorum causes a wide range of clinical manifestations in dogs, the most frequent being respiratory signs (cough, dyspnoea), bleeding disorders (haemorrhages) and neurological symptoms, which may lead to severe or fatal outcomes [13-15]. This variety, in addition to further unspecific clinical signs, can make the diagnosis of canine angiostrongylosis challenging. A specific diagnosis can be reached using the reference standard technique (Baermann method), based on the detection of first-stage larvae (L1) in faecal samples [16]. The morphological identification of $\mathrm{L} 1$ requires expertise, as the larvae can be misidentified as that of other lungworms such as Crenosoma vulpis or Filaroides spp., as well as a variety of free-living nematodes that can contaminate the samples. FLOTAC represents another coproscopic method used for the detection of $A$. vasorum L1 in faecal samples, with a good sensitivity [17]. However, both coproscopic techniques have the disadvantage that they cannot detect prepatent infections. The prepatent period is 38-57 days, when damage to the lung parenchyma is already present $[18,19]$. More recently, specific PCRs [20, 21] and serological methods [22, 23] have been developed for the identification of infected animals. Serological methods (ELISAs) are used for clinical diagnosis of individual cases but also for epidemiological screening [24]. Furthermore, a rapid commercial blood test is available for the detection of A. vasorum antigens in domestic dogs (IDEXX Angio Detect ${ }^{\mathrm{TM}}$, IDEXX Laboratories, Westbrook, ME, USA) with a sensitivity of $84.6 \%$ in clinically suspect dogs [25].

In Romania, the current occurrence of A. vasorum in domestic and wild canids is poorly known. The parasite was identified by necropsy in $4.2 \%$ of the red foxes from the western part of the country [26]. Larval stages resembling A. vasorum have been reported in faeces from dogs from the Timiș County, but they were not molecularly confirmed and the origin and travel history of the dogs was not specified [27]. So far, no studies have been conducted to assess the prevalence and distribution of $A$. vasorum in domestic dogs in Romania and the awareness among vets is limited or absent (Mihalca, personal communication).

The aim of the present study was to evaluate the countrywide seroprevalence of specific circulating antigens of $A$. vasorum and specific antibodies against $A$. vasorum in domestic dogs from Romania.

\section{Methods}

Between November 2016 and July 2017, a total of 1545 domestic dogs from 23 counties of Romania were included in the study. Details about their gender, age, breed, housing, use and origin were collected (full data and categories used for statistical analysis are given in Additional file 1) for each dog. Details about the previous anthelmintic treatments were also collected, but due to the largely incomplete dataset on this point (no information on the date of the last treatment, no information on the product used, etc.) this was excluded from the data analysis. Blood samples (5-9 ml) were collected from the cephalic vein using S-Monovette $9 \mathrm{ml}$, Clotting Activator/Serum (Sarstedt, Nümbrecht, Germany). The serum was separated by centrifugation and stored at $-20{ }^{\circ} \mathrm{C}$ until use. All samples were tested at the Institute of Parasitology, Vetsuisse Faculty, University of Zurich, Switzerland, for the presence of $A$. vasorum circulating antigens (AG) using monoclonal and polyclonal antibodies in a sandwich ELISA, with a sensitivity of $95.7 \%$ and a specificity of $94.0 \%$, as described by Schnyder et al. [22]. Additionally, a sandwich ELISA (sensitivity $81.0 \%$, specificity 98.8\%) using A. vasorum adult somatic antigen purified by monoclonal antibodies (mAb Av 5/5) was used for specific antibody (AB) detection [23]. Test thresholds were regionally determined based on the mean value of optical density $\left(\mathrm{A}_{405} \mathrm{~nm}\right)$ plus three standard deviations of 300 randomly selected samples [28]. All test runs included a background control, a conjugate control, three positive control sera from three experimentally infected dogs and two negative control sera from uninfected dogs.

The collected data were analysed using EpiInfo ${ }^{\mathrm{TM}} 7$ software (CDC, Atlanta, GA, USA). The prevalence of $\mathrm{AB}$ and/or AG presence and corresponding 95\% confidence intervals $(95 \% \mathrm{CI})$ were calculated. Differences among the various categories were assessed by means of Chisquare testing and were considered statistically significant for values with $P<0.05$. 


\section{Results}

A total of 33 dogs (2.14\%, 95\% CI: $1.82-3.56 \%)$ were seropositive for $A$. vasorum antigen or antibodies against the parasite. Three dogs were positive for antigen only (0.19\%, 95\% CI: $0.07-0.57 \%)$ and 30 dogs (1.94\%, 95\% CI: 1.36-2.76\%) were positive for antibodies only. No dog was positive for both tests. The prevalence by county (AG or $\mathrm{AB}$ ) varied between 1.61 and $6.06 \%$ (Table 1, Fig. 1). The prevalence for each dog category and statistical data are shown in Additional file 2.

The overall prevalence ( $\mathrm{AB}$ or $\mathrm{AG}$ ) and the $\mathrm{AB}$ prevalence were significantly higher in pure breed dogs compared to mixed breeds and mongrel dogs $\left(\chi^{2}=6.264\right.$, $d f=2, P=0.043$ for $\mathrm{AB}$ and $\chi^{2}=6.677, d f=2, P=0.035$ for $A B$ or $A G$ ). Among service groups (use), the overall prevalence $(\mathrm{AB}$ or $\mathrm{AG})$ and the $\mathrm{AB}$ prevalence were significantly higher in shepherd dogs compared to other groups $\left(\chi^{2}=10.463, d f=4, P=0.033\right.$ for $\mathrm{AB}$ and $X^{2}=10.401, d f=4, P=0.034$ for $\mathrm{AB}$ or $\left.\mathrm{AG}\right)$. When considering only the $A B$ prevalence, there was a significant difference between counties $\left(\chi^{2}=34.32, d f=22, P=0.045\right)$. However, when considering the overall prevalence $(\mathrm{AB}$ or AG), there was no significant difference between counties. Moreover, there was no significant difference between males and females, between urban and rural dogs, between dogs with unrestricted access and with restricted access to the environment, and between dogs living outdoors and indoors. The statistical analyses indicated a significantly higher overall ( $A B$ or $A G$ ) prevalence and $A B$ prevalence in dogs aged below 6 months $\left(\chi^{2}=11.537, d f=3, P=0.009\right.$ for $\mathrm{AB}$ and $\chi^{2}=11.043$, $d f=3, P=0.011$ for $\mathrm{AB}$ or $\mathrm{AG}$ ).

\section{Discussion}

Similar studies using the same diagnostic approach were performed in several countries across Europe (Table 2). The main difference so far is that Romania is the only country (except Bulgaria, where the sample size was small) where no dog was positive for both $\mathrm{AG}$ and $\mathrm{AB}$ detection. Generally, the percentage of such dogs was very low $(0.28 \%$ in Italy to $1.36 \%$ in Hungary). Similarly to the case of Romania, most of the positive dogs included in the previous studies were positive only for the antibodies $(0.56 \%$ in Italy to $3.11 \%$ in Slovakia). According to Schnyder et al. [25, 28, 29], the seropositivity only to antibodies may indicate a

Table 1 Seroprevalence of A. vasorum circulating antigens and antibodies by county in dogs from Romania $(n=1545)$

\begin{tabular}{|c|c|c|c|c|c|c|c|}
\hline \multirow[t]{2}{*}{ County } & \multirow[t]{2}{*}{ Examined } & \multicolumn{2}{|l|}{$A G$} & \multicolumn{2}{|l|}{$A B$} & \multicolumn{2}{|c|}{$A G$ or $A B$} \\
\hline & & $\%$ & $95 \% \mathrm{Cl}$ & $\%$ & $95 \% \mathrm{Cl}$ & $\%$ & $95 \% \mathrm{Cl}$ \\
\hline Hunedoara & 165 & 0 & $0-2.21$ & 6.06 & $2.94-10.86$ & 6.06 & $2.94-10.86$ \\
\hline Harghita & 52 & 0 & $0-6.85$ & 5.77 & $1.21-15.95$ & 5.77 & $1.21-15.95$ \\
\hline Satu Mare & 45 & 0 & $0-7.87$ & 4.44 & $0.54-15.15$ & 4.44 & $0.54-15.15$ \\
\hline Maramureș & 123 & 0.81 & $0.02-4.45$ & 3.25 & $0.89-8.12$ & 4.07 & $1.33-9.23$ \\
\hline Mehedinți & 88 & 1.14 & $0.03-6.17$ & 2.27 & $0.28-7.97$ & 3.41 & $0.71-9.64$ \\
\hline Constanța & 32 & 0 & $0-10.89$ & 3.13 & $0.08-16.22$ & 3.13 & $0.08-16.22$ \\
\hline Vrancea & 35 & 2.86 & $0.07-14.92$ & 0 & $0-10.00$ & 2.86 & $0.07-14.92$ \\
\hline Timiș & 141 & 0 & $0-2.58$ & 2.13 & $0.44-6.09$ & 2.13 & $0.44-6.09$ \\
\hline Bihor & 95 & 0 & $0-3.81$ & 2.11 & $0.26-7.40$ & 2.11 & $0.26-7.40$ \\
\hline Mureș & 102 & 0 & $0-3.55$ & 1.96 & $0.24-6.90$ & 1.96 & $0.24-6.90$ \\
\hline Vâlcea & 62 & 0 & $0-5.78$ & 1.61 & $0.04-8.66$ & 1.61 & $0.04-8.66$ \\
\hline Arad & 75 & 0 & $0-4.80$ & 0 & $0-4.80$ & 0 & $0-4.80$ \\
\hline Bacău & 20 & 0 & $0-16.84$ & 0 & $0-16.84$ & 0 & $0-16.84$ \\
\hline Braşov & 39 & 0 & $0-9.03$ & 0 & $0-9.03$ & 0 & $0-9.03$ \\
\hline București & 128 & 0 & $0-2.84$ & 0 & $0-2.84$ & 0 & $0-2.84$ \\
\hline Caraș-Severin & 111 & 0 & $0-3.27$ & 0 & $0-3.27$ & 0 & $0-3.27$ \\
\hline Cluj & 2 & 0 & $0-84.19$ & 0 & $0-84.19$ & 0 & $0-84.19$ \\
\hline Dolj & 35 & 0 & $0-10.00$ & 0 & $0-10.00$ & 0 & $0-10.00$ \\
\hline Galați & 55 & 0 & $0-6.85$ & 0 & $0-6.85$ & 0 & $0-6.85$ \\
\hline lași & 32 & 0 & $0-10.89$ & 0 & $0-10.89$ & 0 & $0-10.89$ \\
\hline Sălaj & 43 & 0 & $0-8.22$ & 0 & $0-8.22$ & 0 & $0-8.22$ \\
\hline Suceava & 22 & 0 & $0-15.44$ & 0 & $0-15.44$ & 0 & $0-15.44$ \\
\hline Tulcea & 43 & 0 & $0-8.22$ & 0 & $0-8.22$ & 0 & $0-8.22$ \\
\hline Total & 1545 & 0.19 & $0.07-0.57$ & 1.94 & $1.36-2.76$ & 2.14 & $1.82-3.56$ \\
\hline
\end{tabular}




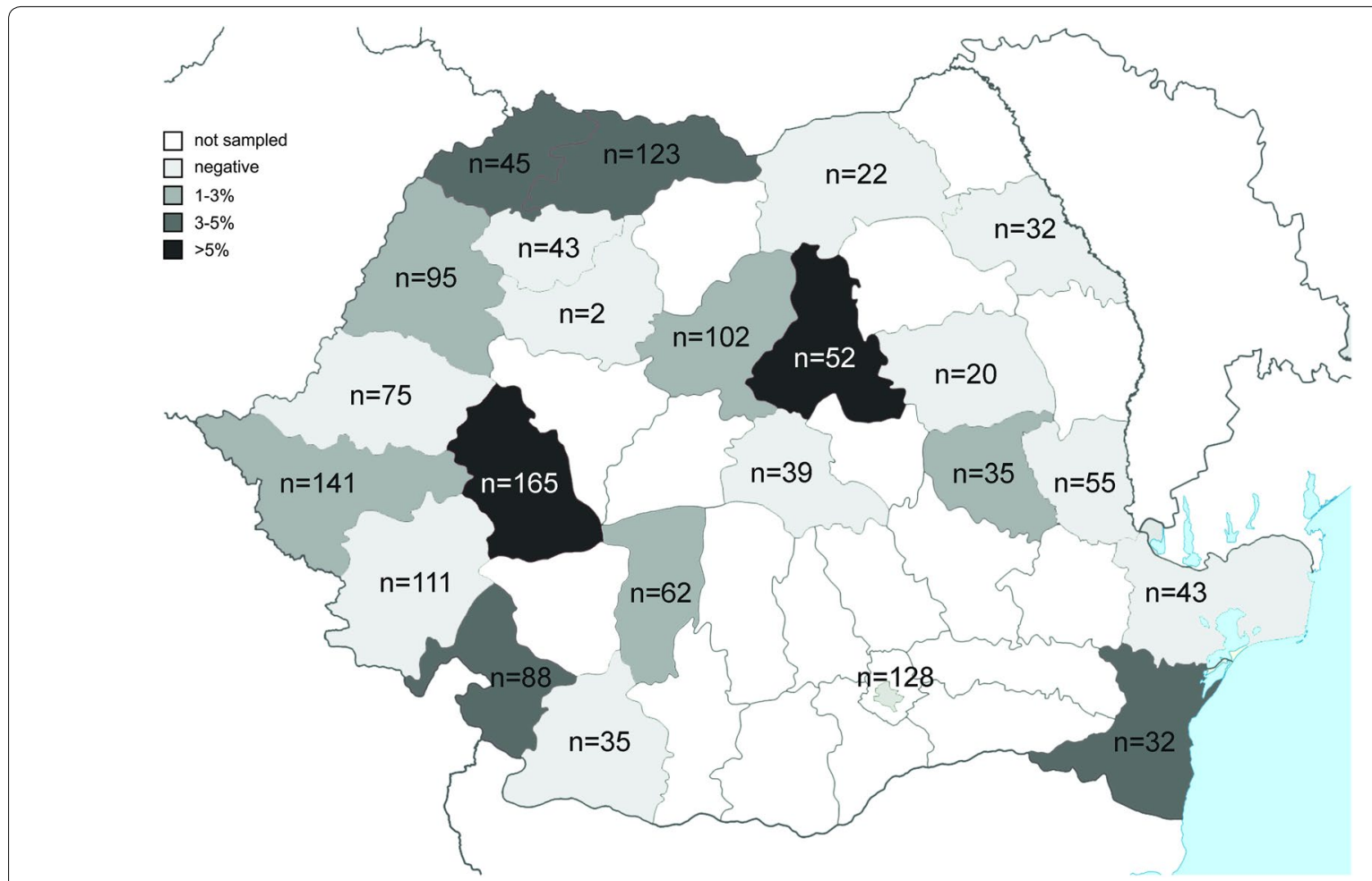

Fig. 1 Seroprevalence of A. vasorum circulating antigens or antibodies by county. The number of sampled dogs is indicated for each county

Table 2 Overview of the serological results for A. vasorum tests performed in Europe

\begin{tabular}{|c|c|c|c|c|c|c|}
\hline Country & Sample size & $A B$ and $A G(\%)$ & AB only (\%) & AG only (\%) & $A G$ or $A B(\%)$ & Reference \\
\hline Romania & 1545 & $0(0.00)$ & $30(1.94)$ & $3(0.19)$ & $33(2.14)$ & Present study \\
\hline Germany & 4003 & $13(0.32)$ & $77(1.92)$ & $7(0.17)$ & $97(2.42)$ & {$[28]$} \\
\hline UK & 4030 & $39(0.97)$ & $90(2.23)$ & $14(0.35)$ & $143(3.55)$ & [28] \\
\hline Poland & 3345 & $17(0.51)$ & $43(1.29)$ & $26(0.78)$ & $86(2.57)$ & [37] \\
\hline Italy & 712 & $2(0.28)$ & $4(0.56)$ & $0(0)$ & $6(0.84)$ & [24] \\
\hline Hungary & 1247 & $17(1.36)$ & 34 (2.73) & $22(1.76)$ & $73(5.85)$ & [33] \\
\hline Switzerland & 6136 & $59(0.96)$ & $130(2.12)$ & $74(1.21)$ & $263(4.29)$ & [38] \\
\hline Slovakia & 225 & $4(1.10)$ & $7(3.11)$ & $4(1.78)$ & $14(6.22)$ & [39] \\
\hline Bulgaria & 150 & $0(0)$ & $1(0.67)$ & $0(0)$ & $1(0.67)$ & {$[40]$} \\
\hline Portugal & 906 & $6(0.66)$ & $12(1.32)$ & 18 (1.99) & $36(3.97)$ & [41] \\
\hline France & 2289 & $26(1.14)$ & $46(2.01)$ & $14(0.61)$ & $86(3.76)$ & {$[42]$} \\
\hline Sweden & 3885 & $4(0.10)$ & $34(0.88)$ & $20(0.51)$ & $58(1.49)$ & {$[43]$} \\
\hline
\end{tabular}

parasite exposure, meaning that the sampling took place (i) during the prepatency (i.e. between 3 and 5 weeks post-infection) when circulating antigens are not yet detectable (detection starts between 5 and 11 weeks post-infection); (ii) after death of parasites following an anthelmintic treatment; or (iii) natural clearance of the infection, as in these cases it may take 3-7 weeks and 3-9 weeks for negative $A G$ and $A B$ results, respectively.
This explains the higher number of dogs testing positive for antibody detection compared to circulating antigen detection [28]. However, as false positive and false negative results may occur in both ELISAs, the positive predictive value is highest when obtaining positive results for both $A G$ and $A B$ detection [28]. This is particularly important in areas with an expected low prevalence, such as in the present case. 
The dog-related risk factors for $A$. vasorum infection were reviewed on several occasions and exhaustively discussed by Morgan et al. [30]. Generally, age is considered a risk factor, with dogs under 18 months showing a higher risk of infection. Other factors such as the month of diagnosing the infection, or sex were found not to influence the risk of infection [30], as in our study.

Based on the literature, in recent years the number of reports both in wild canids and domestic dogs has increased in Europe. This might represent either a true emergence and/or an increased awareness and surveillance by using recently developed diagnostic procedures. However, such assumptions are not possible for countries where surveillance and/or routine testing were historically absent, as in the case of Romania. Several elements (i.e. climate, definitive and intermediate host density) were incriminated as predictive emergence drivers leading to increased risk for canine A. vasorum infections [31]. According to this model, western Romania falls within a low eco-climatic index and the eastern part is within the zero-risk area. However, the authors do not exclude smaller disease foci under favourable microclimatic conditions [31]. It has been previously summarised that the prevalence in dog populations is lower than in fox populations from the same area [32]. Recently, the western part of Romania has been surveyed for the presence of $A$. vasorum in red foxes, Vulpes vulpes [26]. The overall prevalence was $4.2 \%$, which was considered at the lower limit compared to other European countries.

Little is known about the presence of $A$. vasorum in the countries surrounding Romania. According to the available epidemiological data, Romania is located at the eastern margin of the geographical distribution of canine angiostrongylosis: on the western side, the presence of A. vasorum was confirmed in dogs [33], foxes [34] and golden jackals (Canis aureus) [35] from Hungary, while south-eastwards $A$. vasorum was detected in dogs [36] and a golden jackal in Serbia [4].

\section{Conclusions}

The present survey provides a useful update on the epidemiological situation of $A$. vasorum in dogs from Romania. The detection of circulating antigens and/or specific antibodies against $A$. vasorum demonstrates the presence of this parasite in domestic dogs in Romania, in addition to the recent report in foxes [26]. However, so far, no clinical cases have been reported in the country and this may be related to a low awareness among vets. The situation in the eastern half of the country and beyond remains poorly surveyed, and, also based on the absence of dogs seropositive for both antigen and antibody detection, more studies in foxes and domestic dogs are required. Awareness campaigns among vets and owners are essential in order to prevent fatal cases of canine angiostrongylosis.

\section{Additional files}

Additional file 1: Table S1. Raw dataset including the information for all dogs included in the study.

Additional file 2. Detailed values for all calculated statistical values.

\section{Acknowledgements}

We give special thanks to Bayer Animal Health Romania (Alina Georgiana Constantinescu) and Bayer Animal Health GmbH for kindly providing the funds for the laboratory work of DG. We would like to thank the following people for their invaluable help during the sampling: Alexandru Sarmași, "Antonia Save Animals" Association and Antonia Kertesz, Ramona Chirilă, Laura Chira, Nora and Florin Gaciu, Solyom Levente, Cătălin Ionescu, Cristian Pascu, Adrian Cioran, Ganea Dorin Cristian, Viorica (Baia Mare), Magos Attila, Balog Iosif, Danyflor Dog Shelter, Robert Popa and Ioana Pădurean, Claudiu Zurgalău, Oachiș Agnes, Gîscă Dan,"Prietenii Animalelor" Association, Dombay Endre, Jakab Agatha, Ana-Maria Diacu and Lorena Paraschiva.

\section{Authors' contributions}

GD designed the study, collected samples, analysed the samples and wrote the manuscript; NGG analysed the samples; AMI collected samples and performed the statistical analysis; AM, IRP, CDC, MI, ILM, CR, DB, MN and RO collected samples; VC supervised the PhD work of GD; RL wrote parts of the text; MS coordinated the laboratory work, supervised the work and co-designed the study; and ADM wrote part of the manuscript, co-designed the study, collected samples and coordinated sample collection. All authors critically revised the manuscript. All authors read and approved the final manuscript.

\section{Funding}

Funding was provided by Bayer Animal Health Romania and Bayer Animal Health $\mathrm{GmbH}$. This project is funded by the Ministry of Research and Innovation of Romania, Projects for Financing the Excellence in CDI, Contract no. 37PFE/06.11.2018

\section{Availability of data and materials}

The datasets used and/or analysed during the present study are available in the additional files associated with this manuscript.

Ethics approval and consent to participate

The study was done according to national and European ethical regulations.

\section{Consent for publication}

Not applicable.

\section{Competing interests}

The authors declare that they have no competing interests.

\section{Publisher's Note}

Springer Nature remains neutral with regard to jurisdictional claims in published maps and institutional affiliations.

\section{Author details \\ ${ }^{1}$ Department of Parasitology and Parasitic Diseases, University of Agricultural Sciences and Veterinary Medicine Cluj-Napoca, Cluj-Napoca, Romania. ${ }^{2}$ Insti- tute of Parasitology, Vetsuisse-Faculty, University of Zurich, Zurich, Switzerland. ${ }^{3}$ Department of Parasitology and Parasitic Diseases \& Animal Biology, Faculty of Veterinary Medicine, University of Agronomic Sciences and Veterinary Medicine of Bucharest, Bucharest, Romania. ${ }^{4}$ Department of Public Health, Faculty of Veterinary Medicine, University of Agricultural Sciences and Veteri- nary Medicine, Iaşi, Romania. ${ }^{5}$ Federal Research Institute for Animal Health, Institute of Infectology, Friedrich-Loeffler-Institute, Riems, Germany. ${ }^{6}$ SC Veterra SRL, Sighişoara, Romania. ${ }^{7}$ Bayer Animal Health GmbH, 51368 Lev- erkusen, Germany.}

Received: 7 January 2019 Accepted: 3 May 2019

Published online: 14 May 2019 


\section{References}

1. Morgan ER, Shaw SE, Brennan SF, De Waal TD, Jones BR, Mulcahy G. Angiostrongylus vasorum: a real heartbreaker. Trends Parasitol. 2005;21:49-51.

2. Magi M, Guardone L, Dell'omodarme M, Prati M, Mignone W, Torracca B, Monni G, Macchioni F. Angiostrongylus vasorum in red foxes (Vulpes vulpes) and badgers (Meles meles) from Central and Northern Italy. Hystrix It J Mamm. 2010;20:121-6.

3. Hermosilla C, Kleinertz S, Silva LM, Hirzmann J, Huber D, Kusak J, Taubert A. Protozoan and helminth parasite fauna of free-living Croatian wild wolves (Canis lupus) analyzed by scat collection. Vet Parasitol. 2017:233:14-9.

4. Gavrilović P, Marinković D, Todorović I, Gavrilović A. First report of pneumonia caused by Angiostrongylus vasorum in a golden jackal. Acta Parasitol. 2017;62:880-4.

5. Bourque A, Whitney $\mathrm{H}$, Conboy G. Angiostrongylus vasorum infection in a coyote (Canis latrans) from Newfoundland and Labrador, Canada. J Wildl Dis. 2005;41:816-9.

6. Bertelsen MF, Meyland-Smith F, Willesen $J$, Jefferies R, Morgan ER, Monrad J. Diversity and prevalence of metastrongyloid nematodes infecting the red panda (Ailurus fulgens) in European zoos. Vet Parasitol. 2010;172:299-304.

7. Gillis-Germitsch N, Manser MB, Hilbe M, Schnyder M. Meerkats (Suricata suricatta), a new definitive host of the canid nematode Angiostrongylus vasorum. Int J Parasitol Parasites Wildl. 2017;6:349-53.

8. Guilhon J. Recherches sur le cycle évolutif du Strongle des vaisseaux du chien. Bull Acad Vét. 1963;36:431-42.

9. Rosen L, Ash LR, Wallace GD. Life history of the canine lungworm Angiostrongylus vasorum (Baillet). Am J Vet Res. 1970;31:131-43.

10. Mozzer LR, Lima WS. Gallus gallus domesticus: paratenic host of Angiostrongylus vasorum. Vet Parasitol. 2015;207:81-4.

11. Barçante TA, de Paiva Barçante JM, Dias SR, dos Santos Lima W. Angiostrongylus vasorum (Baillet, 1866) Kamensky, 1905: emergence of thirdstage larvae from infected Biomphalaria glabrata snails. Parasitol Res. 2003;91:471-5.

12. Serres E. Entozoaires trouvés dans l'oreille droite, le ventricule correspondant et l'artère pulmonaire d'un chien. J Vét Midi. 1854;7:70.

13. Helm JR, Morgan ER, Jackson MW, Wotton P, Bell R. Canine angiostrongylosis: an emerging disease in Europe. J Vet Emerg Crit Care. 2010;20:98-109.

14. Staebler S, Ochs H, Steffen F, Naegeli F, Borel N, Sieber-Ruckstuhl N, Deplazes P. Autochthone Infektionen mit Angiostrongylus vasorum be Hunden in der Schweiz und Deutschland. Schweiz Arch Tierheilkd. 2005:147:121-7.

15. Sigrist NE, Hofer-Inteeworn N, Jud Schefer R, Kuemmerle-Fraune C, Schnyder M, Kutter AP. Hyperfibrinolysis and hypofibrinogenemia diagnosed with rotational thromboelastometry in dogs naturally infected with Angiostrongylus vasorum. J Vet Intern Med. 2017:31:1091-9.

16. Deplazes P, Eckert J, Mathis A, von Samson-Himmelstjerna G, Zahner H. Parasitology in veterinary medicine. Wageningen: Wageningen Academic Publishers; 2016.

17. Schnyder M, Maurelli MP, Morgoglione ME, Kohler L, Deplazes P, Torgerson P, Cringoli G, Rinaldi L. Comparison of faecal techniques including FLOTAC for copromicroscopic detection of first stage larvae of Angiostrongylus vasorum. Parasitol Res. 2011;109:63-9.

18. Guilhon J, Cens B. Migrations and development of Angiostrongylus vasorum (Baillet, 1866) in the dog. C R Acad Sci Hebd Séances Acad Sci D. 1969;269:2377-80 (In French)

19. Schnyder M, Fahrion A, Ossent P, Kohler L, Webster P, Heine J, Deplazes P. Larvicidal effect of imidacloprid/moxidectin spot-on solution in dogs experimentally inoculated with Angiostrongylus vasorum. Vet Parasitol. 2009; 166:326-32.

20. Jefferies $R$, Morgan ER, Shaw SE. A SYBR green real-time PCR assay for the detection of the nematode Angiostrongylus vasorum in definitive and intermediate hosts. Vet Parasitol. 2009;166:112-8.

21. Al-Sabi MN, Deplazes P, Webster P, Willesen JL, Davidson RK, Kapel CM. PCR detection of Angiostrongylus vasorum in faecal samples of dogs and foxes. Parasitol Res. 2010;107:135-40.

22. Schnyder M, Tanner I, Webster P, Barutzki D, Deplazes P. An ELISA for sensitive and specific detection of circulating antigen of Angiostrongylus vasorum in serum samples of naturally and experimentally infected dogs. Vet Parasitol. 2011:179:152-8.
23. Schucan A, Schnyder M, Tanner I, Barutzki D, Traversa D, Deplazes P. Detection of specific antibodies in dogs infected with Angiostrongylus vasorum. Vet Parasitol. 2012:185:216-24.

24. Guardone L, Schnyder M, Macchioni F, Deplazes P, Magi M. Serological detection of circulating Angiostrongylus vasorum antigen and specific antibodies in dogs from central and northern Italy. Vet Parasitol. 2013;192:192-8.

25. Schnyder M, Stebler K, Naucke TJ, Lorentz S, Deplazes P. Evaluation of a rapid device for serological in-clinic diagnosis of canine angiostrongylosis. Parasit Vectors. 2014;7:72.

26. Deak G, Gherman CM, lonică AM, Vezendan AD, D'Amico G, Matei IA, et al. Angiostrongylus vasorum in Romania: an extensive survey in red foxes, Vulpes vulpes. Parasit Vectors. 2017;10:330.

27. Ilie MS, Imre M, Imre K, Hora FȘ, Dărăbuș G, Jurca AO, et al. Prevalence of Angiostrongylus vasorum infestation in dogs from western Romania-preliminary results. Lucr Șt USABT Med Vet. 2016;49:78-81.

28. Schnyder M, Schaper R, Bilbrough G, Morgan ER, Deplazes P. Seroepidemiological survey for canine angiostrongylosis in dogs from Germany and the UK using combined detection of Angiostrongylus vasorum antigen and specific antibodies. Parasitology. 2013;140:1442-50.

29. Schnyder M, Jefferies R, Schucan A, Morgan ER, Deplazes P. Comparison of coprological, immunological and molecular methods for the detection of dogs infected with Angiostrongylus vasorum before and after anthelmintic treatment. Parasitology. 2015;142:1270-7.

30. Morgan ER, Jefferies R, Van Otterdijk L, McEniry RB, Allen F, Bakewell M, Shaw SE. Angiostrongylus vasorum infection in dogs: presentation and risk factors. Vet Parasitol. 2010;173:255-61.

31. Morgan ER, Jefferies R, Krajewski M, Ward P, Shaw SE. Canine pulmonary angiostrongylosis: the influence of climate on parasite distribution. Parasitol Int. 2009;58:406-10.

32. Koch J, Willesen JL. Canine pulmonary angiostrongylosis: an update. Vet J. 2009:179:348-59.

33. Schnyder M, Schaper R, Lukács Z, Hornok S, Farkas R. Combined serological detection of circulating Angiostrongylus vasorum antigen and parasite-specific antibodies in dogs from Hungary. Parasitol Res. 2015;114(Suppl. 1):145-54.

34. Sréter T, Széll Z, Marucci G, Pozio E, Varga I. Extraintestinal nematode infections of red foxes (Vulpes vulpes) in Hungary. Vet Parasitol. 2003:115:329-34

35. Takács A, Szabó L, Juhász L, Takács A, Lanszki J, Takácz P, Heltai M. Data on the parasitological status of golden jackal (Canis aureus L., 1758) in Hungary. Acta Vet Hung. 2013;62:33-41.

36. Simin S, Kosić LS, Kuruca L, Pavlović I, Savović M, Lalošević V. Moving the boundaries to the South-East: first record of autochthonous Angiostrongylus vasorum infection in a dog in Vojvodina province, northern Serbia. Parasit Vectors. 2014;7:396.

37. Schnyder M, Schaper R, Pantchev N, Kowalska D, Szwedko A, Deplazes P. Serological detection of circulating Angiostrongylus vasorum antigen and parasite-specific antibodies in dogs from Poland. Parasitol Res. 2013;112(Suppl. 1):109-17.

38. Lurati L, Deplazes P, Hegglin D, Schnyder M. Seroepidemiological survey and spatial analysis of the occurrence of Angiostrongylus vasorum in Swiss dogs in relation to biogeographic aspects. Vet Parasitol. 2015;212:219-26.

39. Miterpáková M, Schnyder M, Schaper R, Hurníková Z, Čabanová V. Serological survey for canine angiostrongylosis in Slovakia. Helminthologia. 2015:52:205-10.

40. Pantchev N, Schnyder M, Vrhovec MG, Schaper R, Tsachev I. Current surveys of the seroprevalence of Borrelia burgdorferi, Ehrlichia canis, Anaplasma phagocytophilum, Leishmania infantum, Babesia canis, Angiostrongylus vasorum and Dirofilaria immitis in dogs in Bulgaria. Parasitol Res. 2015:114:117-30.

41. Alho AM, Schnyder M, Schaper R, Meireles J, Belo S, Deplazes P, de Carvalho LM. Seroprevalence of circulating Angiostrongylus vasorum antigen and parasite-specific antibodies in dogs from Portugal. Parasitol Res. 2016;115:2567-72.

42. Schnyder M, Bilbrough G, Hafner C, Schaper R. Angiostrongylus vasorum, "The French Heartworm": a serological survey in dogs from France introduced by a brief historical review. Parasitol Res. 2017;116:31-40.

43. Grandi G, Osterman-Lind E, Schaper R, Schnyder M. Canine angiostrongylosis in Sweden: a summary of five-year diagnostic activity (2011-2015) and a nationwide seroepidemiological survey using combined detection of specific antibodies and circulating antigens by ELISAs. Acta Vet Scandinava. 2017:59:85. 